\title{
Influence of Pressure on Free Radical Decay in Irradiated Poly(ethylene terephthalate)
}

\author{
F. Szö́cs and T. LACZA \\ Polymer Institute of the Slovak Academy of Sciences, \\ 80934 Bratislava, Czechoslovakia. \\ (Received February 18, 1975) \\ KEY WORDS: Poly(ethylene terephthalate) / Irradiation / Free- \\ Radical Decay / ESR / $\gamma$-Rays /
}

Poly(ethylene terephthalate) (PET) Arnite produced AKZO Holland ( $\mathrm{mp} 250^{\circ} \mathrm{C}$ in vacuo) was hot-pressed into a pellet $(6 \phi \times 6 \mathrm{~mm})$ at $250^{\circ} \mathrm{C}$, $500 \mathrm{~atm}$, and cooled in $45 \mathrm{~min}$. The pellet was irradiated with gamma-radiation for $4.6 \mathrm{Mrad}$ at $-50^{\circ} \mathrm{C}$ and the initial concentration of free radicals was determined by the ESR method. The pellet was then put into the pressing equipment ${ }^{1,3}$, subjected to pressure, and heated to a constant temperature. The annealing was interrupted by a rapid cooling of the pressing equipment; the pellet was then put back into the ESR spectrometer to estimate the resulting concentration of free radicals. The rate constant of free radical decay was determined from the initial and resulting concentrations of the free radicals.

The ESR spectrum of free radicals after irradiation is shown in Figure 1A. The shape of the spectrum changes during the decay of the free radicals. Figure $1 \mathrm{~B}$ shows the spectrum after $20 \mathrm{~min}$ annealing at $3000 \mathrm{~atm}$ and $80^{\circ} \mathrm{C}$, Figure 1C after $20 \mathrm{~min}$ annealing at $1000 \mathrm{~atm}$

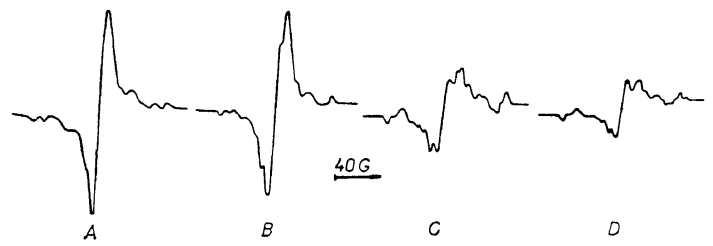

Figure 1. ESR spectra of irradiated poly(ethylene terephthalate) at room temperature: $\mathrm{A}$, immediately after irradiation; B, after $20-\mathrm{min}$ annealing at $80^{\circ}$ and $3000 \mathrm{~atm} ; \mathrm{C}$, after $20-\mathrm{min}$ annealing at $70^{\circ}$ and $1000 \mathrm{~atm} ; \mathrm{D}$, after $20-\mathrm{min}$ annealing at $115^{\circ}$ and $3000 \mathrm{~atm}$. and $70^{\circ} \mathrm{C}$, and Figure $1 \mathrm{D}$ after $20 \mathrm{~min}$ annealing at $3000 \mathrm{~atm}$ and $115^{\circ} \mathrm{C}$. We do not intend to study the problem of the interpretation of these spectra. We shall just limit ourselves to the determination of the total concentration of free radicals.

The decay of free radicals obeys second-order kinetics (Figure 2) and we determined the rate constants of free-radical decay on this basis. The rate constant at $50^{\circ} \mathrm{C}$ depends on pressure only a little (Figure 3). The slope of the individual curves in the temperature region from $50^{\circ} \mathrm{C}$ to $80^{\circ} \mathrm{C}$ increases with temperature. In the pressure range from 2000 to $5000 \mathrm{~atm}$ and at temperatures between $50^{\circ} \mathrm{C}$ and $80^{\circ} \mathrm{C}$ the rate constant depends only slightly on pressure. With increasing pressure the free volume of the polymer decreases, especially in the amorphous

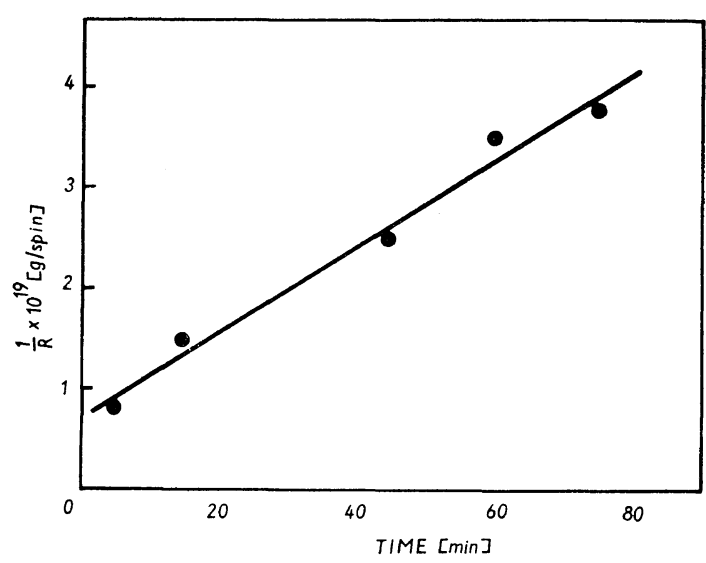

Figure 2. Reciprocal of the free-radical concentration as a function of time at $80^{\circ} \mathrm{C}$ and $4000 \mathrm{~atm}$. 


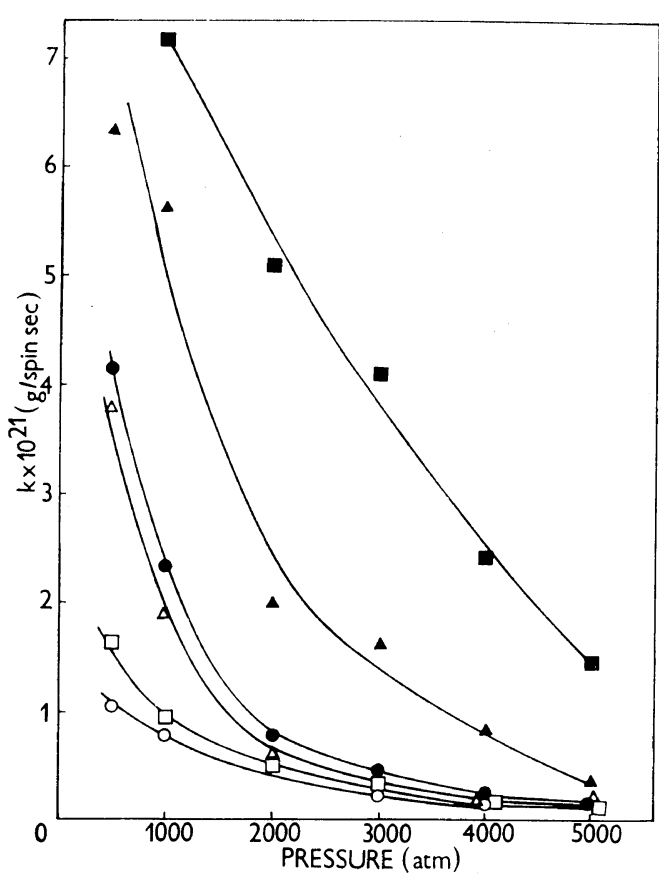

Figure 3. Rate constants of the free-radical decay as a function of pressure at different temperatures: $\mathrm{O}, 50^{\circ} \mathrm{C} ; \square, 60^{\circ} \mathrm{C} ; \triangle, 70^{\circ} \mathrm{C} ; \bullet, 80^{\circ} \mathrm{C} ; \triangle, 100^{\circ} \mathrm{C}$; a, $115^{\circ} \mathrm{C}$.

regions; because of this, the molecular motion which allows the decay of free radicals is hindered. Further increase in pressure in the region from 2000 to $5000 \mathrm{~atm}$ does not affect the rate of decrease any more because the segmental motion that brings about the intensive decay has already been suppressed. The pressure dependence of the rate constant at $105^{\circ} \mathrm{C}$ shows a quite different character. The radicals which occur predominantly in the crystalline regions of the polymer decay at high temperatures. The dependence is almost linear, due to the mechanism of the pressure effect on the molecular motion in the crystalline phase. The molecular motion in the crystalline regions of the polymer is reduced proportionally to the pressure; no saturation appears, as in the amorphous phase where the free volume of the polymer decreases rapidly.

If we compare our results with those obtained from mechanical relaxations, a nice correspondence can be seen. Illers and Breuer ${ }^{2}$ studied

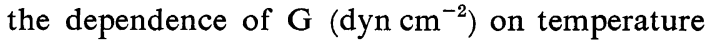
of PET upon varying the degree of crystallinity. Their results showed a narrow maximum at about $80^{\circ} \mathrm{C}$ for amorphous samples $(0-2.5 \%$ of crystallinity) and a broad maximum at about $100^{\circ} \mathrm{C}$ for crystalline samples $(33-46 \%$ of crystallinity). In principle, the results of our experiments are in good agreement with the course of the loss curve of the mechanical $\alpha$-relaxation. The rate constant of decay is practically independent of pressure until the temperature reaches the value corresponding to the $\alpha$-relaxation region. In the neighborhood of the maximum of the $\alpha$-relaxations of the amorphous regions approximately $80^{\circ} \mathrm{C}$ ) we can observe a remarkable effect of pressure on the free radical decay occurring in the amorphous regions. At higher temperatures (above $100^{\circ} \mathrm{C}$ ) the pressure dependence of the rate constant is almost linear and its value is relatively high in the region of higher pressures (3000-4000 atm). At higher pressures and temperatures, free radicals decay mainly in the crystalline regions which are less sensitive to the effects of pressure than the amorphous regions.

\section{REFERENCES}

1. F. Szöcs, J. Appl. Polym. Sci., 14, 2629 (1970).

2. K. H. Illers and H. Breuer, J. Colloid Sci., 18, 1 (1963).

3. F. Szöcs, Proceedings of the Third Tihany Symposium on Radiation Chemistry, Publishing House of the Hungarian Academy of Sciences, Bundapest, 1972, p 933. 